\title{
Antibacterial Activity of Nanostructured Polyaniline Combined With Mupirocin
}

\author{
Kaveeta Pergas Jotiram ${ }^{1,2}$, R. G. S. V. Prasad ${ }^{1 *}$, Venkata Srinivas Jakka ${ }^{1}$, R.S.L.Aparna ${ }^{1}$ and A.R. Phani ${ }^{3}$ \\ ${ }^{1}$ School of Pharmacy, Masterskill University College of Health Science, Kualalumpur, 43200, Malaysia \\ ${ }^{2}$ Faculty of science, technology and engineering La' Trobe University, Bendigo Victoria 3552, Australia \\ ${ }^{3}$ Nano-Research for Advanced Materials and Technologies, Bangalore, 560040, Karnataka, India \\ * Corresponding author: gunnaeswara79@gmail.com Tel.: 0838943435
}

\begin{abstract}
Nanostructured and conducting polymer polyaniline (PANI) is used in numerous applications in electrotherapy, electro-magnetic materials for monitoring health, antimicrobial clothing, data transfer in smart textiles, biosensors and for defense technology. An important criterion for all the above mentioned utilities is, producing polymeric conductive fibers. In the present study we prepared conducting PANI nanofibres combined with mupirocin, a topical antimicrobial agent, through a self-assembly process. The prepared polymer was then tested for the antibacterial properties against various Gram positive and Gram negative bacteria such as Streptococcus pyogenes, Staphylococcus epidermidis, Staphylococcus aureus and Escherichia coli. Scanning electron microscopy (SEM) and Fourier transform infrared spectroscopy (FTIR) were used to identify the chemical structure of the PANI nanofibres. The antibacterial properties were assessed by measuring the zones of inhibition. It was evident from these results that antimicrobial activity increased with increasing PANI and PANI combined with mupirocin (PANI-mupirocin) concentrations. It was also found that PANI-mupirocin has enhanced antimicrobial activity compared to PANI alone. This information might be useful to evaluate the potential use of nanostructured polyaniline in fabrics incorporated with antibacterial agents as a prophylactic use against bacterial skin infections in the near future.
\end{abstract}

Keywords: Nanostructured polyaniline, Mupirocin, Antimicrobial activity

Citation: K.P. Jotiram, et al. Antibacterial Activity of Nanostructured Polyaniline Combined With Mupirocin. Nano Biomed. Eng. 2012, 4(3), 144-149. DOI: 10.5101/nbe.v4i3. p144-149.

\section{Introduction}

Research and development in the nanotechnology field is growing rapidly throughout the world, and there is no surprise that it is expected to be the basis of many of the main technological innovations of the 21 st century. The development of new materials in the nanometre scale such as nanoparticles is a major breakthrough in the nanotechnology field. Nanoparticles are defined as particulate materials with at least one dimension of less than 100 nanometres (nm), even the particles could be of zero dimensions as in the case of quantum dots. These include carbon nanotubes, semiconductor quantum dots, metal nanowires and other nanoparticles produced from a huge variety of substances.

With nanotechnology and nanoparticles becoming increasingly prominent in daily life, recent advances in these fields have created great opportunity to revolutionize the textile industry. An increasing improvement in the qualitative standards of human lifestyles as well has brought to the invention of "smart fabrics". Smart fabrics are intelligent or smart textiles that have the ability to react and adapt to stimuli sensed from the environment by integration of functionalities in the textile structure. The stimulus as well as the response can be electrical, thermal, chemical, magnetic or other origin. The reason why smart fabrics have attracted interest throughout the world is because of their ability to be used in applications such as antimicrobial clothing [1], electrotherapy [2], health monitoring shirts [3], transferring of data in clothing, military applications [4] and biosensors [5].

Attempts of grafting polymers onto the surface of fabrics have been made by scientists in the development of smart textiles. Polymers are favored here accounting to their light weight and greater ease of fabrication. Polyaniline (PANI) [6], polypyrroles (PPy), polythiophenes (PT) and polyphenylene vinylenes (PPV) are examples of polymers used in smart fabrics. In the article "Conducting Polymers" by S.Ramakrishnan, the author has stressed that among these polymers, 
polyaniline stands out compared to the other polymers, the reason being it is the only polymer that can be doped by a protic acid and can exist in different forms depending upon the $\mathrm{pH}$ of the medium. Nanostructured and conducting polyaniline is also used in the application of smart fabrics because of its high conductivity and sensitivity to chemical vapors. Other reasons why polyaniline is also favoured to be used in the application of smart fabrics would include its low production cost, its ability to show high thermal and chemical stability in the conductive form and it can easily be doped with organic and inorganic acids [7].

When it comes to a wide range of bacterial invaders, the skin is able to present a first line defence against these invaders. However when the skin's natural defence weakens accidentally or intentionally, the role for antibacterials emerge. Most antibacterials used topically offer several advantages, such as avoidance of systemic toxicity leading to fewer side effects, decreased induction of bacterial resistance, and the high concentration of antibacterial agent at the site of infection [8]. One good example is mupirocin (pseudomonic acid A), an antibiotic produced by Pseudomonas fluorescens which has been shown to possess high activity against staphylococci, streptococci, and certain Gram-negative bacteria such as Neisseria gonorrhoeae and Haemophilus influenza [9].

Its clinical use has been limited to topical preparations, commonly available as a $2 \%$ ointment, due to its high protein binding and marked reduction of its antibacterial activity in the presence of human serum [9]. Its mechanism of action is by inhibiting bacterial protein synthesis by specific reversible binding to bacterial isoleucyl tRNA synthase [10].

In the present study we prepared conducting PANI nanofibres combined with mupirocin (PANI-mupirocin) through a self-assembly sol-gel process. The prepared was then evaluated for the antibacterial properties against various Gram positive and Gram negative bacterial Streptococcus pyogenes, Staphylococcus epidermidis, Staphylococcus aureus and Escherichia coli. Viours methods were also be used to identify the structure and chemical nature of the PANI nanofibres. This information might be useful to evaluate the potential use of nanostructured polyaniline fabrics incorporated with antibacterial agents as a prophylactic use against bacterial skin infections in the near future.

\section{EXPERIMENTAL DETAILS}

\subsection{Chemicals:}

Aniline, ammonium persulfate (APS), camphor sulphonic acid (CSA) and mupirocin obtained from Sigma Aldrich with $99.5 \%$ purity. Methanol, barium chloride, sulphuric acid, acetone and dimethlysulfoxide were reagent grade. Sabouraud agar and Nutrient broth were obtained from HiMedia.

\subsection{Preparation of PANI and PANI-mupirocin}

Nano Biomed. Eng. 2012, 4(3),144-149
Aniline monomer was transferred into the $100 \mathrm{~mL}$ beaker and $10 \mathrm{~mL}$ of distilled water was added and the beaker is kept in ice bath. The contents of the beaker were stirred for $5 \mathrm{~min}$. Required quantity of camphorsulfonic acid was weighed in a $100 \mathrm{~mL}$ beaker and $10 \mathrm{~mL}$ of distilled water was added and the contents were stirred for $5 \mathrm{~min}$ till it dissolved in water. The contents of the beaker containing the surfactant (CSA) were transferred to the monomer dropwise by using a funnel and the total contents were stirred for $5 \mathrm{~min}$. Then APS was weighed in a $100 \mathrm{~mL}$ beaker and $10 \mathrm{~mL}$ of distilled water was added to it and the contents were stirred for 5 min till it was dissolved in water. After the addition of surfactant, the contents of the beaker containing the oxidant (APS) were added to the monomer contents dropwise. The final contents were stirred for about 10 min and the contents were kept in freezer operating at 0 ${ }^{\circ} \mathrm{C}$ for 24 hours. The contents were removed and filtered by washing several times with double distilled water till the unreacted surfactant is washed. The green polyaniline solid was dried over night in oven at $100{ }^{\circ} \mathrm{C}$. To prepare PANI-mupirocin, $25 \mathrm{mg}$ of mupirocin was weighed and dissolved in $25 \mathrm{~mL}$ of distilled water and mixed with CAS solution and the same method was followed. Therefore, 1 $\mu \mathrm{g}$ PANI-mupirocin contained $0.05 \mu \mathrm{g}$ mupirocin.

\subsection{Antibacterial activity test for PANI nano- fibers}

To carry out the antibacterial activity for PANI nanofibres, nutrient agar medium was prepared by using peptone $(5.0 \mathrm{~g})$, beef extract $(3.0 \mathrm{~g})$, and sodium chloride $(\mathrm{NaCl})(5.0 \mathrm{~g})$ in $1000 \mathrm{~mL}$ distilled water and the $\mathrm{pH}$ was adjusted to 7.0 and agar $(15.0 \mathrm{~g})$ was added to the solution. The agar medium was sterilized in aquilots of 15 $\mathrm{mL}$ at a pressure of $15 \mathrm{lbs}$ for $15 \mathrm{~min}$. This nutrient agar medium was transferred into sterilized petri dishes in a laminar air flow unit. After solidification of the media, a $24 \mathrm{hr}$ culture of each organism was standardized to 0.5 McFarland standard culture, which was then cultivated as a lawn culture on the solid surface of the media. Cup plate method was used to test the antibacterial activity by using sterile bore with the diameter of $9 \mathrm{~mm}$. To this petri dish, one drop of respective dilution of PANI in dimethylsulfoxide solution (DMSO) $(5,10,15,20$ and $30 \mu \mathrm{g} \mathrm{mL}^{-1}$ ) was added using micropipette and incubated for 2 days at $37{ }^{\circ} \mathrm{C}$ in the incubation chamber. Average zone diameters were measured using Intech antibiotic zone reader (model IN -1215, India).All the experiments were done in triplicate and average values were taken into account.

\subsection{Antibacterial activity test for PANI-mupiro- cin nanofibers}

To carry out the antibacterial activity for PANImupirocin nanofibres, nutrient agar medium prepared by using peptone $(5.0 \mathrm{~g})$, beef extract $(3.0 \mathrm{~g})$, and sodium chloride $(\mathrm{NaCl})(5.0 \mathrm{~g})$ in $1000 \mathrm{ml}$ distilled water 


\section{RESULTS}

\subsection{Morphological Properties}

\subsubsection{Scanning Electron Microscopy (SEM)}

The prepared particles of PANI-mupirocin nanofibers were studied by SEM (Hitachi S800, Japan). A fixed working distance of $5 \mathrm{~mm}$ and a voltage of $5-25 \mathrm{kV}$ were used. It was found that the reaction temperature and to some extent aging temperature and time strongly affect the microstructure and the formation probability of PANImupirocin nanofibers.In all the cases we have obtained nanofiber like structures but with different lengths and diameter. The SEM image of PANI-mupirocin nanofibers which shown in Fig. 1 which indicates the nanofiber diameter about $10 \mathrm{~nm}$. And also the nanofibers are hollow in nature. The magnifications of the sample were reported in order of $\mathrm{a}, \mathrm{b}$ and $\mathrm{c}$.

\subsection{Structural Characterization}

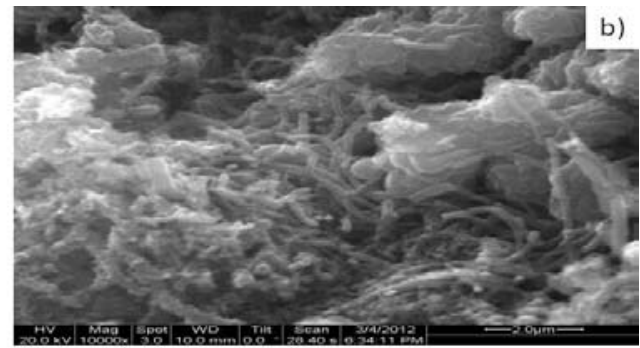

c)

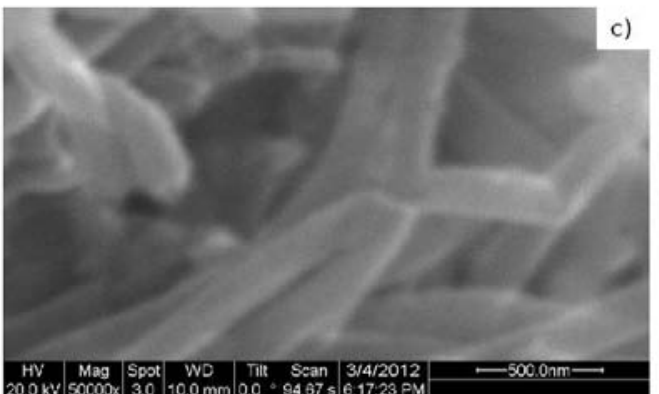

Fig. 1 SEM of prepared PANI-mupirocin was shown at a magnification of (a) 5000; (b) 10000; (c) 50000

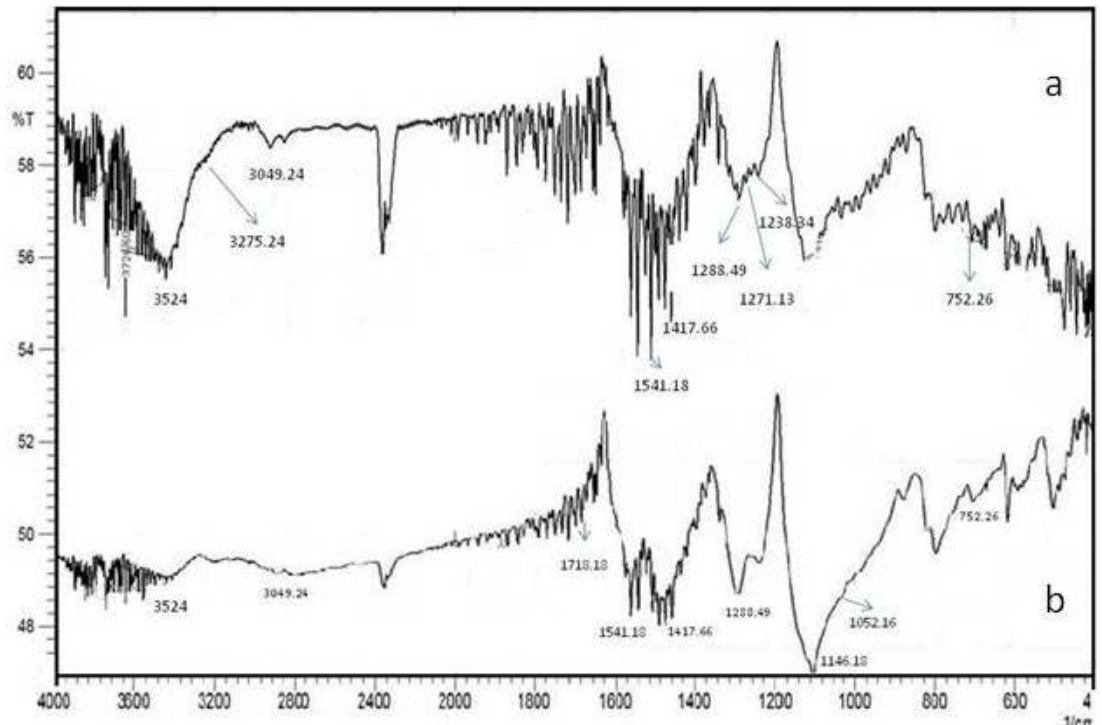

Fig. 2 FTIR spectra of (a) PANI nanofibres (b) PANI-mupirocin nanofibers 


\subsubsection{Fourier transform infrared spectroscopy (FTIR)}

FTIR (model 3116465, shimadzu, Japan) spectrum of pure PANI (Fig. 2a) shows peaks at 1541 and $1485 \mathrm{~cm}^{-1}$ shows the presence of quinoid and benzenoid ring. This clearly indicates the respective two states in the polymer chain. Several other peaks at $1176 \mathrm{~cm}^{-1}$ are due to the stretching vibration of $\mathrm{C}-\mathrm{N}$ bond, $752 \mathrm{~cm}^{-1}$ indicates out-of-plane deformation of $\mathrm{C}-\mathrm{H}$ on monosubtituted or 1,2-disubtituted ring, $1238 \mathrm{~cm}-1$ stretching $\mathrm{C}-\mathrm{N}$ bond in benzenoid ring, $1271 \mathrm{~cm}^{-1}$ and $1288 \mathrm{~cm}^{-1}$ respectively shows the presence of $\mathrm{C}-\mathrm{N}$ bond on primary and secondary amine, $1417 \mathrm{~cm}^{-1}$ due to phenazine ring stretching, $3049 \mathrm{~cm}^{-1}$ due to aromatic ring stretching of $\mathrm{C}-\mathrm{H}$, and $3275 \mathrm{~cm}^{-1}$ due to $\mathrm{H}$ bonded stretching of $\mathrm{N}-\mathrm{H}$. All this values are characteristic of polyaniline chain. Fig. 2b showed FTIR spectrum of PANI-mupirocin. There are characteristic peaks of PANI and principal peaks mupirocin of at 1718,1146 and $1052 \mathrm{~cm}^{-1}$. This concludes that a blend of polymer mixture was formed with antibiotic mupirocin.

\subsection{Antimicrobial activity}

Both PANI and PANI-mupirocin showed considerable antibacterial activity on all the bacterial tested. As the concentration of PANI and PANI-mupirocin increased, the zone of inhibition also increased. Among all the species tested, it was Streptococcus pyogenes that showed the highest average value for zone of inhibition, followed by Staphylococcus epidermidis, Staphylococcus aureus and finally Escherichia coli. Fig. 3 shows zones of inhibitions of PANI and PANI-mupirocin on Staphylococcus epidermidis (NCIM-2604). Control DMSO showed no inhibitory zones. Zone of inhibitions were observed as 5, 5.67, 6, 6.3 and $7.3 \mathrm{~mm}$ for corresponding PANI concentrations of 5,10,15,20 and $30 \mu \mathrm{g} \mathrm{mL}^{-1}$, respectively. Inhibitory concentrations for PANI-mupirocin were noted as $5.3 \mathrm{~mm}$ at $5 \mu \mathrm{g} \mathrm{mL}^{-1}, 6.3 \mathrm{~mm}$ at $10 \mu \mathrm{g} \mathrm{mL}^{-1}, 7 \mathrm{~mm}$ at $15 \mu \mathrm{g} \mathrm{mL}^{-1}, 7.67 \mathrm{~mm}$ at $20 \mu \mathrm{g} \mathrm{mL}^{-1}$ and $8.67 \mathrm{~mm}$ at 30 $\mu \mathrm{g} \mathrm{mL}{ }^{-1}$. From this it was evident that the minimum zone of inhibition of PANI and PANI-mupirocin for $E$. coli (ATCC-25922) was $5 \mu \mathrm{g} \mathrm{mL}^{-1}$. For $S$. pyogenes (NCIM2608). PANI showed zone of inhibitions of $9 \mathrm{~mm}$ for concentration $5 \mu \mathrm{g} \mathrm{mL} \mathrm{m}^{-1}, 9.3 \mathrm{~mm}$ for concentration of PANI at $10 \mu \mathrm{g} \mathrm{mL}^{-1}, 10 \mathrm{~mm}$ for concentration of PANI at $15 \mu \mathrm{g} \mathrm{mL}^{-1}, 10.67 \mathrm{~mm}$ at $20 \mu \mathrm{g} \mathrm{mL}^{-1}$ and $11 \mathrm{~mm}$ at 30 $\mu \mathrm{g} \mathrm{mL}{ }^{-1}$. Inhibitory concentrations for PANI-mupirocin were noted as $9.3 \mathrm{~mm}$ at $5 \mu \mathrm{g} \mathrm{mL}^{-1}, 9.67 \mathrm{~mm}$ at $10 \mu \mathrm{g}$ $\mathrm{mL}^{-1}, 10.3 \mathrm{~mm}$ at $15 \mu \mathrm{g} \mathrm{mL}^{-1}, 11 \mathrm{~mm}$ at $20 \mu \mathrm{g} \mathrm{mL}^{-1}$ and $11.67 \mathrm{~mm}$ at $30 \mu \mathrm{g} \mathrm{mL}^{-1}$. These results confirm that there is clear enhancement of antibacterial activity when PANI combined with mupirocin.

Fig. 4a shows the antibacterial activity of PANI and PANI-mupirocin against Streptococcus pyogenes (NCIM-2608). Both PANI and PANI-mupirocin showed considerable antibacterial activity on all the concentrations tested, however PAN-mupirocin showed larger zones of inhibition, which signifies better antibacterial activity. Streptococcus pyogenes (NCIM-2608) is more susceptible with their average zone diameters noted highest at concentrations of $30 \mu \mathrm{g} \mathrm{mL}^{-1}$ for both PANI and PANImupirocin. The difference in average zone of inhibition diameter for PANI and PANI-mupirocin was also noted to be greatest at $30 \mu \mathrm{g} \mathrm{mL}^{-1}$ which was measured to be $0.67 \mathrm{~mm}$. The difference in average zone of inhibition diameter for concentrations of 5, 10, 15 and $20 \mu \mathrm{g} \mathrm{mL}^{-1}$ were measured to be almost similar, ranging from 0.30 $\mathrm{mm}$ to $0.37 \mathrm{~mm}$. Fig. $4 \mathrm{~b}$ shows the antibacterial activity of PANI and PANI-mupirocin against Staphylococcus aureus (NCIM-2079). As the concentrations increased, so did the zones of diameters. Staphylococcus aureus (NCIM-2079) was more succeptable with their average zone diameters noted highest at average zone diameters noted highest at concentrations of $30 \mu \mathrm{g} \mathrm{mL} \mathrm{m}^{-1}$ for both PANI and PANI-mupirocin. The difference in average zone of inhibition diameter for PANI and PANImupirocin was also noted to be greatest at $20 \mu \mathrm{g} \mathrm{mL}^{-1}$ which measured $0.37 \mathrm{~mm}$. The difference in average zone of inhibition diameter for concentrations of 5, 10, 15 and $30 \mu \mathrm{g} \mathrm{mL}^{-1}$ were measured to be almost similar, ranging from $0.30 \mathrm{~mm}$ to $0.34 \mathrm{~mm}$. Fig. 4c shows the antibacterial activity of PANI and PANI combined mupirocin against Escherichia coli (ATCC-25922). The difference in average zones of inhibition for PANI and PANI-mupirocin was also noted to be greatest at 20 and $30 \mu \mathrm{g} \mathrm{mL}^{-1}$ which measured $1.37 \mathrm{~mm}$. There was a steady increase in the inhibition diameter for concentrations of 5,10 and $15 \mu \mathrm{g} \mathrm{mL}^{-1}$, ranging from $0.30 \mathrm{~mm}$ to 1.00 mm. Fig. 4d shows the antibacterial activity of PANI and PANI-mupirocin against Staphylococcus epidermidis (NCIM-2604). As the concentrations increased, so did the zones of diameters. The difference in the average zones of inhibition diameter for PANI and PANI-mupirocin was also noted to be greatest at 5, 20 and $30 \mu \mathrm{g} \mathrm{mL}{ }^{-1}$ which measured $0.67 \mathrm{~mm}$. Based on the above discussion, it is evident that PANI-mupirocin has an enhanced activity on Streptococcus pyogenes (NCIM-2608), Staphylococcus epidermidis (NCIM-2604), Staphylococcus aureus (NCIM-2079) and Escherichia coli (ATCC-25922) compared to PANI alone. Streptococcus pyogenes (NCIM-2608) showed a good susceptibility to mupirocin [11].

The ability of PANI and its derivatives to inhibit various bacteria were reported. The actual mechanism by which PANI exhibits antibacterial activity is by triggering oxidative damage-responsive genes and suppressing the formation of bacterial cell walls $[12,13]$. So PANI and mupirocin can potentially inhibit bacterial growth by two different mechanisms providing enhanced antibacterial activity of the composite.

\section{CONCLUSION}

In this investigation, we have synthesized nanofibres of PANI and PANI-mupirocin by a simple wet chemical template-free method. The obtained nanofibers of PANI and PANI-mupirocin were characterized by 
http://nanobe.org

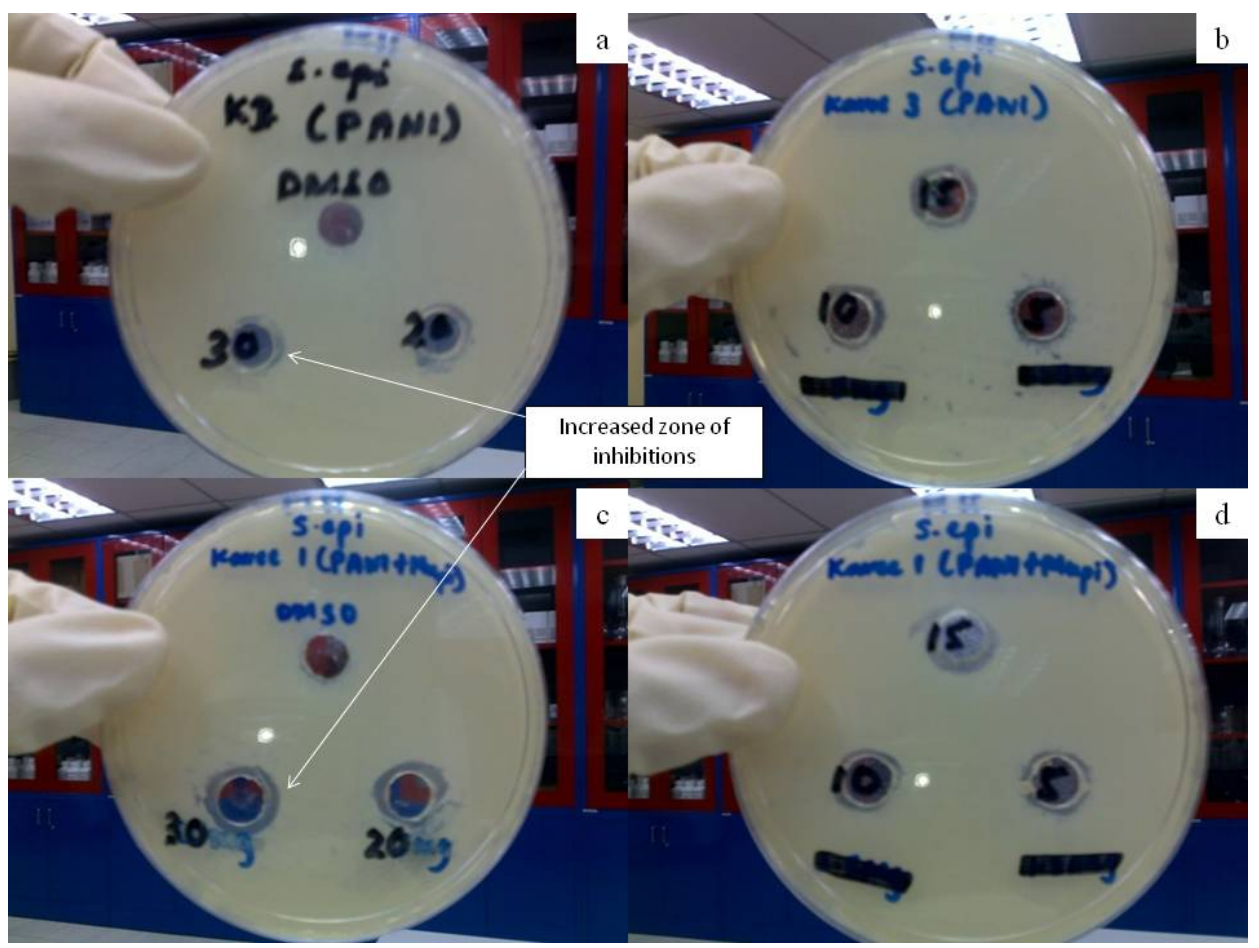

Fig. 3 Photographic image of zones of inhibition at various concentrations of PANI and PANI-mupirocin on Staphylococcus epidermidis (NCIM-2604). The antibacterial activity of PANI on Staphylococcus epidermidis (NCIM2604). (a) Control DMSO and PANI at 30 and $20 \mu \mathrm{g} \mathrm{mL}^{-1}$. (b) PANI at 15,10 and $5 \mu \mathrm{g} \mathrm{mL}^{-1}$. (c) Control DMSO, PANImupirocin at 30 and $20 \mu \mathrm{g} \mathrm{mL}^{-1}$. (d) PANI-mupirocin at 15,10 and $5 \mu \mathrm{g} \mathrm{mL}$
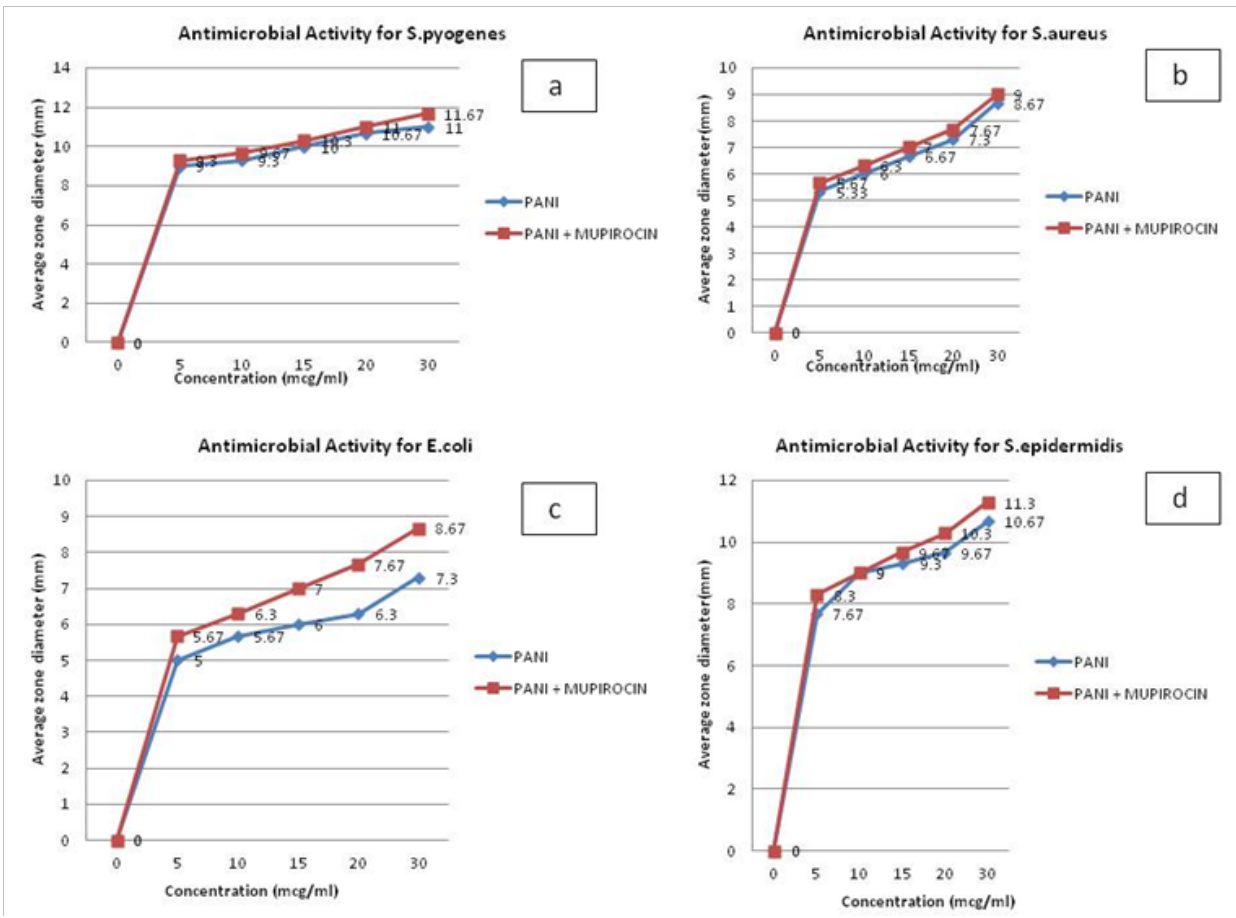

Fig. 4 Antimicrobial activity of PANI and PANI-mupirocin: S. pyogenes (a); S. aureus (b); E. coli (c); S. epidermidis (d)

standard techniques where it has been confirmed that PANI structure is formed. The PANI-mupirocin in DMSO under different concentrations have shown excellent antimicrobial activity on various bacterial tested. The different concentration of PANI-mupirocin showed enhanced activity on the bacteria compared to concentrations of PANI alone. Among all the four bacteria, it was Streptococcus pyogenes (NCIM-2608) which showed the greatest zone of inhibition for both
PANI and PANI-mupirocin concentrations. These studies will have a great potential application in the designing of smart clothing like antibacterial fabrics which will serve in prophylactic use where polyaniline is one of the ingredients.

\section{Acknowledgements}

The authors are grateful to the management of the Masterskill university of health science, Malaysia for promoting research and 
providing financial support in carrying out this investigation and NanoRAM Technologies,Bangalore, Karnataka State, India for their technical support.

\section{References}

1 P. Xue, K.H. Park, X.M. Tao, W. Chen and X.Y. Cheng. Electrically conductive yarns based on PVA/carbon nanotubes. Compos. Struct. 2007; 78: 271-277.http://dx.doi.org/10.1016/j.compstruct.2005. 10.016

2 K.W. Oh, H.J. Park, S.H. Kim,Stretchable conductive fabric for electrotherapy. Appl. Polym. Sci. 2003;88:1225-1229. http://dx.doi. org/10.1002/app.11783

3 D. Bowman and B. R. Mattes, Conductive fibre prepared from ultrahigh molecular weight polyaniline for smart fabric and interactive textile applications.Synth. Met. 2005; 154: 29-32. http://dx.doi.org/ 10.1016/j.synthmet.2005.07.017

4 C. Y. Lee, H. G. Song, K. S. Jang, E. J. Oh, A. J. Epstein and J. Joo, Electromagnetic interference shielding efficiency of polyaniline mixtures and multilayer films.Synth. Met. 1999;102: 1346-1349. http://dx.doi.org/10.1016/S0379-6779(98)00234-3

5 Z. M. Tahir, E. C. Alocilja and D. L. Grooms,Polyaniline synthesis and its biosensor application.Biosens bioelectro.2005;15:1690-1695. http://dx.doi.org/10.1016/j.bios.2004.08.008

6 K. W. Oh, S. H. Kim and E. A. Kim,Improved surface characteristics and the conductivity of polyaniline-nylon 6 fabrics by plasma treatment. J Appl Polym Sci. 2001;81:684-694. http://dx.doi.org/10.1002/ app. 1485

7 M. R. Karim, J.H. Yeum, M.S. Lee,K.T.Lim,Preparation of conducting polyaniline/ $\mathrm{TiO}_{2}$ composite submicron-rods by the gammaradiolysis oxidative polymerization method. React Funct Polym. 2008; 68:1371-1376. http://dx.doi.org/10.1016/j.reactfunctpolym. 2008.06.016 http://nanobe.org

8 C. Gelmetti,Local antibiotics in dermatology. Dermatol Therapy. 2008;21:187-195.http://dx.doi.org/10.1111/j.1529-019.2008.00190.x

9 B. D. Cookson, The emergence of mupirocin resistance: a challenge to infection control and antibiotic prescribing practice.J. Antimicrob. Chemother. 1998; 41: 11-18. http://dx.doi.org/10.1093/jac/41.1.11

10 P. J. O'Hanlon, N. H. Rogers, J. W. Tyler,The chemistry of pseudomonic acid. Part 6. Structure and preparation of pseudomonic acid D. J. Chem. Soc, Perk. T 1. 1983; 2655-2657.http://dx.doi.org/ 10.1039/P19830002655

11 M. Kresken, Prevalence of mupirocin resistance in clinical isolates of Staphylococcus aureus and Staphylococcus epidermidis: results of the Antimicrobial Resistance Surveillance Study of the PaulEhrlich-Society for Chemotherapy, 2001. Int. J. Antimicrob. Ag. 2004; 23: 577-581.http://dx.doi.org/10.1016/j.ijantimicag.2003. 11.007

12 R. Marija, G. Nikolaidis, J. R. Bennett, S. Swift, A. J. Easteal and M. Ambrose,Broad spectrum antimicrobial activity of functionalized polyanilines. Acta Biomaterialia. 2011; 7: 4204-4209.http://dx.doi. org/10.1016/j.actbio.2011.07.018

13 R.G.S. V. Prasad, K.S.V. Chaitanya, M. Tejoram, D. Basavaraju, K.N.Rao, R.R. Kumar, S. Sreenivasan, A.R. Phani, Antibacteria Properties of Nanofiber Structured Conducting Polyaniline Synthesized by Cost Effective Wet Chemical Process J. Pharm. Res. 2012; 5: 370 .

Copyright:(c) 2012 K.P. Jotiram, et al. This is an openaccess article distributed under the terms of the Creative Commons Attribution License, which permits unrestricted use, distribution, and reproduction in any medium, provided the original author and source are credited. 